\title{
SPINOZA Y LA INDIGNACIÓN: UNA CONCEPCIÓN EVOLUCIONISTA DE LA POLÍTICA
}

\section{SPINOZA AND INDIGNATION: AN EVOLUTIONARY CONCEPTION OF POLITICS}

\section{STÉPHANE VINOLO*}

Recibido 4 de julio del 2016 Aceptado 2 de septiembre del 2016 



\title{
SPINOZA Y LA INDIGNACIÓN: UNA CONCEPCIÓN EVOLUCIONISTA DE LA POLÍTICA
}

\section{SPINOZA AND INDIGNATION: AN EVOLUTIONARY CONCEPTION OF POLITICS}

\author{
STÉPHANE VINOLO
}

PALABRAS CLAVE: Afectos, Estado, indignación, mimetismo, René Girard, Spinoza. KEY WORDS: affects, state, indignation, mimicry, René Girard, Spinoza

\begin{abstract}
RESUMEN
El surgimiento del Estado en la filosofía de Spinoza presenta un reto conceptual importante. Dada la ontología espinozista, el orden político no puede provenir de un acuerdo contractual ni de una finalidad antropológica. Mostramos en este trabajo que la única manera de pensarlo es de entender el juego complejo de los afectos y sobre todo de su ley fundamental que es la del mimetismo. Dentro de este juego de los afectos, juego que nace en el estado de la naturaleza pero que sigue al centro de la autoregulación del estado político, es la indignación que, sin duda alguna, mejor cataliza las potencias de los indi-

viduos para crear un Estado y un orden colectivo de manera exclusivamente inmanente. Sin embargo, la indignación tal como la entiende Spinoza, no se basa en valores trascendentes que fundamentan una legitimidad por encima de la legalidad, sino en un puro proceso cuantitativo que nos permite explicar que, tal como vio René Girard, el surgimiento del orden colectivo siempre tiene la forma de un linchamiento. El sistema colectivo que más persevera en su ser es entonces aquel que, sin intenciones ni finalidad de manera exclusivamente evolucionista, logra simular la racionalidad y por lo tanto desplegar toda su potencia.
\end{abstract}




\section{ABSTRACT}

The emergence of the State in the philosophy of Spinoza presents an important conceptual challenge. Given the Spinoza ontology, political order cannot come from a contractual agreement nor from a anthropological purpose. In this article, the author wishes to show that the only way to to think of it is to understand the complex game of of the affects and above all, of its basic law, which is that of mimicry. Within the game of affects, a game that is born in the state of nature but continues to the center of self-regulation of the political state, is indignation that, doubtlessly, catalyses better the power of individuals to create an immanent and exclusive State and collective order. Nevertheless, indignation, as understood by Spinoza, is not founded on trascendental values that collocate legitimacy over legality, but rather on a purely quantitative process that allows us to explain that, as René Girard saw it, the emergence of collective order always has the form of a lynching. The collective system that perseveres more in one's being is then that which without having any intentions nor a purpose exclusively evolutionary, achieves simulating rationality, and thus unfolds all its power.
"Si le désir n'était pas mimétique, nous ne serions ouverts ni à l'humain niau divin. » (Girard, 2001 : 28)

La política de Spinoza presenta una ruptura radical en la Historia de la Filosofía puesto que se basa en una ontología no-finalista e inmanente, que impide cualquier referencia a valores, a contratos o a tendencias. Esta posición filosófica radical cuestiona no solo el tipo de política que se privilegia', sino además la misma definición de la política. Tradicionalmente, el surgimiento del Estado y la necesidad de la política como tal, se basan en una lógica finalista que, utilizando diferentes caminos conceptuales, desde Platón hasta Rawls, obedece a la misma estructura (Matheron, 2011 (2)). En un primer momento de la argumentación, se establecen fines de la naturaleza humana que pueden ser revelados por la razón (en este sentido que quién actúa de manera más racional realiza de la mejor manera esos fines) y se asume que, como estos son los fines de la naturaleza humana, el hombre tiene el deber de perseguirlos, porque de cierta forma, fue creado de tal manera que no puede desear racionalmente otra cosa que realizarlos. Los fines de la naturaleza humana pueden variar según

${ }^{1}$ Recordemos que Spinoza, en pleno siglo XVII y de manera única, afirma el carácter absoluto en todo de la democracia. 
los sistemas filosóficos, pero siempre se determina que el hombre debe perseguir ciertos fines justificándolos en una finalidad ontológica o en una esencialidad humana. Por ejemplo, si la razón nos persuade a perseguir la felicidad en el caso de Aristóteles ${ }^{2}$ y la simple seguridad para poder ir de deseo en deseo en el caso de Hobbes ${ }^{3}$, los dos autores determinan un fin que los seres humanos deben racionalmente perseguir, por lo cual en ambos casos existe un fin de la naturaleza humana que solo nos revela plenamente la razón.

Este primer momento de la argumentación explica el segundo, en el cual se precisa el surgimiento de la política y su carácter necesario. Una vez determinados estos fines, se plantea lógicamente el problema de los medios para alcanzarlos y uno de estos es la creación de un colectivo político, que aparece como un fin condicional que nos permite alcanzar in fine nuestra verdadera finalidad incondicional determinada por la naturaleza humana. Vemos en la Política de Aristó-

\footnotetext{
2 "Es manifiesto, pues, que la felicidad es algo perfecto y suficiente, ya que es el fin de los actos." (Aristóteles, 1988(1), 1097b 20-22: 143)

3 "La felicidad es un continuo progreso de los deseos, de un objeto a otro, ya que la consecución del primero no es otra cosa sino un camino para realizar otro ulterior. La causa de ello es que el objeto de los deseos humanos no es gozar una vez solamente, y por un instante, sino augurar para siempre la vía del deseo futuro." (Hobbes, 1982, I, XI: 84)
}

teles ${ }^{4}$ que la Polis es el tipo de comunidad humana que nos permite realizar nuestro Bien Supremo, que es la felicidad. La Polis es así un medio a través del cual los seres humanos realizan su verdadera finalidad. De la misma manera en Hobbes, el Estado absoluto es la estructura política que mejor permite garantizar el verdadero Bien de todos los seres humanos que es ante todo la seguridad y el sobrevivir para poder ir de deseo en deseo de manera infinita 5 . Así, la política aparece tradicionalmente como una finalidad condicional sobre nuestro camino hacia la realización de otra finalidad que le es superior, por lo que el ser humano cuya vida está dictada por la razón, al no estar rodeado de individuos plenamente racionales, siente el deber de vivir en una comunidad política para poder alcanzar su verdadera finalidad.

Podríamos oponernos a la tradición aristotélica y la tradición hobbe-

4"Puesto que vemos que toda ciudad es una cierta comunidad y que toda comunidad está constituida con miras a algún bien (porque en vista de lo que les parece bueno todos obran en todos sus actos), es evidente que todas tienden a un cierto bien, pero sobre todo tiende al supremo la soberana entre todas y que incluye a todas las demás. Esta es la llamada ciudad y comunidad cívica." (Aristóteles, 1988, I, 1, 1252a: 45-46)

${ }^{5}$ Tal como lo mostró Jean Terrel, la necesidad del Estado surge en Hobbes de un cálculo mínimo según el cual, para poder ir de deseo en deseo de manera infinita, la primera condición es de seguir en vida. Cf. (Terrel, 2001: 135-155) 
siana sobre la manera según la cual se crean las sociedades políticas. En el caso de Aristóteles hay cierta naturalidad en este proceso cuando el pacto introduce el carácter artificial de toda asociación política en Hobbes. No se trata de confundir la diversidad de las teorías filosóficas en cuanto al origen y a la justificación del Estado. Sin embargo y a pesar de estas oposiciones innegables, notemos la común justificación finalista y esencialista de la política que responde a una finalidad fundamental al nivel antropológico. La política es aquí una prótesis para alcanzar un objetivo que le es a la vez exterior y superior.

El mecanismo legitimador de la existencia de la política según la finalidad no puede funcionar en el sistema espinozista, ya que toda finalidad está excluida de la Naturaleza, es decir de la misma divinidad. Más aun, tal como lo vemos en el Apéndice 1 de la primera parte de su Ética, la finalidad es para Spinoza, el origen de todos los errores en contra de los cuales construye su propio sistema filosófico racionalista: "Todos los prejuicios que intento indicar aquí dependen de uno solo, a saber: el hecho que los hombres supongan, comúnmente, que todas las cosas de la naturaleza actúan, al igual que ellos mismos, por razón de un fin e incluso tienen por cierto que Dios mismo dirige todas las cosas hacia un cierto fin..." (Spinoza, 2014, I, Apéndice: 109).
En su proyecto de radicalización de la modernidad que se manifiesta en el rechazo de la finalidad y de las cualidades ocultas a favor de una cuantificación generalizada, Spinoza se ve obligado a proponer otro modelo del surgimiento de la política, así como de su legitimidad, un modelo que no se basa en cierta tendencia humana a la asociación. Pero tampoco puede funcionar el mecanismo contractual, ya que este supone en gran medida hombres capaces de un mínimo de racionalidad y de un cálculo entre costos y beneficios, a raíz del cual van a deducir que es más beneficioso pasar el estado civil que permanecer en la guerra de todos contra todos que manifiesta el estado de naturaleza. Tal como lo ve perfectamente Spinoza, el contrato social supone, para ser posible, que el problema que pretende resolver ya esté resuelto, es decir que los hombres ya decidieron mediatizar sus relaciones con un uso mínimo del logos. Efectivamente, el contrato social solo puede funcionar entre individuos ya capaces y dispuestos a hacer un mínimo de cálculo según los costos y los beneficios que se encuentran entre el renunciar a una parte de su potencia natural a cambio de cierta garantía en términos de seguridad ${ }^{6}$. Supo-

${ }^{6}$ La lógica del contrato supone, además, seres humanos que, en este cálculo, siempre prefieran la vida a la muerte, lo que el terrorismo contemporáneo cuestiona de manera urgente. Cf. (Dupuy, 2002) 
ne animales que ya decidieron renunciar a la violencia inmediata para entrar en el logos. Por lo cual el acuerdo no puede provenir del uso del logos ya que el uso del logos requiere o evidencia, por sí mismo, cierta actitud ética entre los interlocutores.

El error fundamental de los filósofos políticos que preceden a Spinoza, es según este, el establecer un modelo del ser humano (a pesar que este modelo pueda ser muy diferente según los autores) a partir del cual construyeron su política, que solo puede ser válida, por lo tanto, para estos hombres ideales, es decir precisamente para hombres que ya no necesiten la mediación de alguna política ${ }^{7}$. Por este motivo, minimizan lo

\footnotetext{
7 "Los filósofos conciben los afectos, cuyos conflictos soportamos, como vicios en los que caen los hombres por su culpa. Por eso suelen reírse o quejarse de ellos, criticarlos o (quienes quieren aparecer más santos) detestarlos. Y así, creen hacer obra divina y alcanzar la cumbre de la sabiduría, cuando han aprendido a alabar, de diversas formas, una naturaleza humana que no existe en parte alguna y a vituperar con sus dichos lo que realmente existe. En efecto, conciben a los hombres no como son, sino como ellos quisieran que fueran. De ahí que, las más de las veces, hayan escrito una sátira, en vez de una ética y que no hayan ideado jamás una política que pueda llevarse a la práctica, sino otra, que o debería ser considerada como una quimera o solo podría ser instaurada en el país de Utopía o en el siglo dorado de los poetas, es decir, allí donde no hacía falta alguna." (Spinoza, 2013, I, 1: 97-98)
}

más determinante en la mayoría de los seres humanos y lo único que puede servir de etiología política: los afectos.

Para entender los fundamentos de las sociedades humanas, así como para comprender el tipo de necesidad del poder político, es con el único juego de los afectos que debemos proceder a esta explicación; caso contrario, se explicaría una política para hombres imaginarios o estaremos, tal como lo dice Spinoza en varios textos, soñando con los ojos abiertos $^{8}$. Sin embargo, tal como vamos a verlo, el afecto fundamental que permite la creación del orden político en Spinoza, no es un afecto positivo como podrían serlo la generosidad, la fraternidad, la compasión, la abnegación o la simpatía. A diferencia de otros autores, Spinoza nos deja ver que es un afecto sumamente negativo que crea cualquier Estado y que se mantiene en este como su virus autoregulador. Este afecto violento y negativo es la indignación.

Por lo tanto, no podemos limitar el espinozismo a una repetición de los contractualismos en los cuales el Estado aparece como una respuesta racional

8 "Así pues, quienes creen que hablan, o callan, o hacen cualquier cosa, por libre decisión del alma, sueñan con los ojos abiertos.", Spinoza, Ética, III, 2, Escolio, Alianza Editorial, Madrid, 1987, 2014, p. 218. 
a una pasión -el miedo- ${ }^{9}$, ni a un sentimentalismo, en el cual es una pasión positiva que nos conlleva a regular el colectivo humano de manera política, menos aún a un finalismo según el cual tendemos a crear el Estado porque este nos sirve para algo ${ }^{10}$. La política en Spinoza no aparece como conclusión de las pasiones, sino como expresión y relevo de estas; se conserva el juego de los afectos interhumanos desde la creación del Estado hasta su transformación, se pasa por los posibles procesos de revolución, así

${ }^{9}$ De hecho, Spinoza rechaza la ruptura cualitativa del contrato para mantener una continuidad radical entre estado de naturaleza y estado civil, tal como lo vemos en su crítica de Hobbes: "En cuanto atañe a la política, le diferencia entre yo y Hobbes, acerca de la cual usted me consulta, consiste en esto: que yo conservo siempre incólume el derecho natural y afirmo que en cualquier ciudad, a la autoridad suprema no le compete sobre los súbditos un derecho mayor, sino en la medida en que su poder supera al de los súbditos; lo que tiene lugar siempre en el estado natural." (Spinoza, 2007, Carta 50 a Jarig Jelles: 203)

${ }^{10}$ La finalidad aristotélica es, para Spinoza, una cualidad oculta en la cual solo pueden creer las viejezuelas: "No pesa mucho sobre mí la autoridad de Platón, Aristóteles y Sócrates. Me habría sorprendido, si usted hubiese citado a Epicuro, a Demócrito, a Lucrecio o a alguno de los atomistas o defensores de los átomos; pues no ha de sorprendernos que aquellos que inventaron las Cualidades ocultas, las Especies intencionales, las Formas sustanciales y otras mil necedades, hayan fraguado los Espectros y Fantasmas y hayan creído a las viejezuelas para menoscabar la autoridad de Demócrito, de cuya buena fama estaban tan envidiosos que quemaron todos los libros que él había publicado con tanto encomio." (Spinoza, 2007, Carta 56 a Hugo Boxel: 222-223) como el de regulación social. La paradoja que vamos a analizar es entonces que, en Spinoza, el Estado no proviene de la razón para contener las pasiones, sino surge de las pasiones para, in fine, simular un proceso racional.

\section{I - ETIMOLOGÍA AFECTIVA DEL ESTADO}

El problema de los fundamentos del Estado parece recibir dos respuestas radicalmente diferentes en los textos de Spinoza, lo que podría dejar pensar en una contradicción o como mínimo, en una evolución del pensamiento espinozista en cuanto a esta problemática. Muchos de los comentadores de Spinoza proponen una respuesta a la posible evolución de su filosofía política, sobre todo en su relación con el papel de la forma del pacto social, ya que tuvieron que explicar por qué razón, entre el Tratado Teológico-Político y el Tratado Político, el fundamento contractual del poder político desaparece de los textos. Tal como podemos verlo en el capítulo XVI del primero, en este texto Spinoza presenta el surgimiento de la política según la lógica del contrato, lógica que podemos atribuir, en este caso, tanto a una lectura de Hobbes como de Pufendorf. En el Tratado Teológico-Político, Spinoza presenta explícitamente la creación del Estado según un paradigma dialogal y racional: "Por eso debieron establecer, con la máxi- 
ma firmeza y mediante un pacto, dirigirlo [el colectivo humano] todo por el solo dictamen de la razón..." (Spinoza, 2012, XVI, 2: 338). Sin embargo, una dificultad de interpretación de esta problemática surge porque este modelo desaparece completamente del Tratado Político. Es cierto que el término "contrato" todavía cumple cierto papel en el Tratado Político y aparece en varias ocasiones. Primero en el párrafo 6 del capítulo IV: "No cabe duda de que los contratos o las leyes por los que la multitud transfiere su derecho a un Consejo o a un solo hombre deben ser violados, cuando el bien común así lo exige." (Spinoza, 2013, IV, 6: 146). Sin embargo, tal como lo mostró Alexandre Matheron (2011: 206-207), el uso de la palabra contratus en este caso se aplica a la transferencia del derecho (al singular) de la multitud a un Consejo o a un hombre. Por lo que esta transferencia contractual no existe antes de que haya un derecho unificado de la multitud, es decir una relación política.

El contrato es entonces un contrato secundario con el cual se explica cómo una sociedad democrática en la cual rige la multitud, puede transformarse en una sociedad no-democrática. Pero en ningún caso permite explicar cómo la multitud construye su primer derecho que va a transferir, en un segundo momento a entidades políticas. Este uso de la palabra "contrato" no explica la creación de la democracia originaria en la cual los individuos se unen de manera no-contractual. Segundo, se podría objetar a esta desaparición del contrato en el Tratado-Político el hecho que en el párrafo 13 de su segundo capítulo, Spinoza habla explícitamente de un acuerdo: "Si dos se ponen mutuamente de acuerdo y unen sus fuerzas, tienen más poder juntos y, por tanto, también más derecho sobre la naturaleza que cada uno por sí solo." (Spinoza, 2013, II, 13: 116). Más aún, para llevar la objeción lo más lejos posible, notemos que el verbo latín utilizado para pensar este acuerdo es el verbo convenire "Si duo simul conveniant et vires jungant, ..." lo que nos acerca explícitamente de la forma del contrato. Sin embargo, una vez más, Matheron nos permite refutar la interpretación contractualista del verbo convenire ya que la única ocurrencia de este para pensar el surgimiento del Estado en el Tratado Político, está acompañada del adverbio "naturalmente", lo que impide que pensemos este verbo según la lógica del contrato dado que el contrato es un acuerdo artificial: "Dado que los hombres se guían, como hemos dicho, más por la pasión que por la razón, la multitud tiende naturalmente a asociarse, no porque le guíe la razón, sino algún sentimiento común, ..." (Spinoza, 2013, VI, 1: 154). Así, el Tratado Político excluye la posibilidad de una fundación racional del Estado ya 
que la forma contractual solo se da dentro de un colectivo preorganizado por relaciones políticas.

Este punto de la insuficiencia del fundamento contractual en su modalidad racional, se podría notar incluso desde el Tratado Teológico-Político y no únicamente en el abandono de la forma contractual en el Tratado Político, ya que, cuando se evoca explícitamente la hipótesis del contrato en el primero, lo que impide la oposición violenta de los hombres los unos con los otros, no es la fuerza de la razón como tal -como podríamos esperarlo según la lógica del contrato-, sino ya desde entonces un afecto: "Por eso debieron establecer, con la máxima firmeza y mediante un pacto, dirigirlo todo por el solo dictamen de la razón (al que nadie se atreve a oponerse abiertamente por no ser tenido por loco)..." (Spinoza, 2012, XVI, 2: 338) ${ }^{11}$. En esta cita, el fragmento que se ubica dentro de los paréntesis es de igual importancia que el que está localizado fuera. Más aún, solo el paréntesis permite entender el funcionamiento real del contrato y del pacto, ya que podemos preguntarnos ipor qué razón la negociación contractual debería ser pacífica? Todos los académicos saben que las discusiones son violentas, influenciadas por odios personales, por la envidia y la ambición de cada uno. Por

\footnotetext{
${ }^{11}$ [Subrayamos]
}

lo que imaginar que la simple forma del diálogo produzca paz es una ilusión. A un nivel más conceptual, vemos que desde el Tratado Teológico-Político, Spinoza ya percibía que la apuesta del contrato según la cual las normas comunes pueden provenir de un diálogo o de una negociación racional es una ilusión, ya que entrar en diálogo y perseverar en la mediación dialogal de las relaciones humanas, ya supone un primer acuerdo ético ${ }^{12}$.

Spinoza perfectamente ve que la ética no puede provenir del único logos ya que renunciar a la violencia para aceptar entrar en el logos ya es una posición ética mínima. Por lo cual el mismo contrato necesita o una garantía ética positiva o una garantía afectiva negativa. Lo que implica que o el mismo pacto se contradice ya que la pacificación que esperamos de este lo precede como una de sus condiciones de posibilidad (en una posición ética de los negocia-

${ }^{12}$ En este sentido, Spinoza bien ve que la política contractual que supuestamente se aplica a hombres racionales (ya que deben querer negociar), sería paradójicamente inútil para tales individuos: "En efecto, [los filósofos] conciben a los hombres no como son, sino como ellos quisieran que fueran. De ahí que, las más de las veces, hayan escrito una sátira, en vez de una ética y no hayan ideado jamás una política que pueda llevarse a la práctica, sino otra, que o debería ser considerada como una quimera o solo podría ser instaurada en el país de Utopía o en el siglo dorado de los poetas, es decir, allí donde no hacía falta ninguna." (Spinoza, 2013, I, 1: 98). [Subrayamos] 
dores) o hay un afecto negativo que es el verdadero garante de la paz previa a las negociaciones contractuales. Tal como lo vemos en el paréntesis de la cita mencionada, la opción escogida por Spinoza es la del afecto negativo. Es el miedo (o la vergüenza de ser percibido como un loco) que permite la unión racional y que hace que nadie se oponga públicamente a los razonamientos contractuales y que no surja violencia mientras se negocia el contrato (ya sea para no ser ridiculizado o peor aún para no ser violentado por el grupo). Así, incluso cuando Spinoza evoca la hipótesis de un contrato en el Tratado Teológico-Político, es en definitiva un afecto que sirve de regulador social principal y que permite el éxito del modelo del contrato, tal como si el mismo modelo del contrato estuviera sometido desde siempre a la lógica de los afectos.

Pero, sí es un afecto que impide las oposiciones violentas entre los hombres a la hora de los debates y de las negociaciones, la razón -y menos aún cuando no todos los hombres son plenamente racionales-, no basta para garantizar los fundamentos del Estado. Son entonces los afectos y las relaciones interhumanas pasionales que deben servir de etología al Estado y podemos entender en qué medida dado que: "... los hombres están necesariamente sometidos a los afectos." (Spinoza, 2013,
I, 5: 102). Debemos entonces analizar este modelo del surgimiento afectivo del Estado, que surge desde el Tratado Teológico-Político ya que, el mismo modelo del contrato supone ciertos afectos que pueden limitar la oposición de los hombres a la razón y aparece de manera explícita en el Tratado Político, cuando Spinoza explica cuál es el método de su filosofía política y su verdadero objeto de estudio: "...las causas y los fundamentos naturales del Estado no habrá que extraerlos de las enseñanzas de la razón, sino que deben ser deducidos de la naturaleza común de los hombres." (Spinoza, 2013, I, 7: 105).

Para entender la originalidad del pensamiento político de Spinoza, es necesario analizar estos afectos fundamentales que se encuentran en la raíz del Estado, con el fin de evidenciar cuál es el juego de afectos que permite la existencia de un Estado, jerarquizarlos y precisar cuál es el afecto que más contribuye a este proceso. Una vez entendido el afecto catalizador de las potencias, podremos mostrar el proceso exacto que permite justificar la necesidad del Estado, necesidad que no pensaremos como una necesidad finalista según la cual el Estado es necesario para alcanzar cierto fin, sino una necesidad de hecho según la cual, por la estructura de las relaciones interhumanas, el Estado no puede no aparecer, de la misma manera 
que no puede desaparecer completamente ${ }^{13}$.

\section{II- GÉNESIS Y REVOLUCIÓN: LA SOCIEDAD INDIGNADA}

Los afectos que permiten el surgimiento del estado político están expuestos de manera muy clara en el párrafo quinto del primer capítulo del Tratado Político. Spinoza, citando a su Ética, nos permite determinar cuatro afectos fundamentales de la vida afectiva interindividual humana:

Porque es cierto, tal como lo hemos demostrado en nuestra Ética, que los hombres están necesariamente sometidos a los afectos. Y así, por su propia constitución, compadecen a quienes les va mal y envidian a quienes les va bien; están más inclinados a la venganza que a la misericordia; y, además, todo el mundo desea que los demás vivan según su propio criterio y que aprueben lo que uno aprueba y repudien lo que uno repudia. De donde resulta que, como todos desean ser los primeros, llegan a enfrentarse y se esfuerzan cuanto pueden por oprimirse unos a otros; y el

13"De allí que los hombres tienden por naturaleza al estado político, y es imposible que ellos lo destruyan jamás del todo." (Spinoza, 2013, VI, 1: 154) que sale victorioso se gloría más de haber perjudicado a otro que de haberse beneficiado él mismo. (Spinoza, 2013, I, 5: 102-103)

De estos cuatro afectos que nos permiten establecer la etología del estado político, es notable que cada uno de ellos, como era de esperar por su papel genético de las relaciones políticas, implica una relación con cierta alteridad. Los cuatro afectos fundamentales que tienen un impacto en la creación del Estado son la conmiseración, la envidia, la ambición de dominación y el deseo de gloria. Sin embargo, puesto que el mismo Spinoza nos remite a la Ética, el texto citado solo se puede entender a partir de la teoría de la vida afectiva interhumana que yace en las proposiciones 27 a 34 de la tercera parte de este libro. Toda la vida afectiva interhumana se basa en la proposición 27 de la tercera parte de la Ética que establece su ley fundamental, es decir, el carácter mimético de los afectos: "Por el hecho de imaginar que experimenta algún afecto una cosa semejante a nosotros y sobre la cual no hemos proyectado afecto alguno, experimentamos nosotros un afecto semejante." (Spinoza, 2014, III, 27: 241). Esta única ley de la afectividad interhumana explica los cuatro afectos fundamentales, así como sus consecuencias en el estado de naturaleza. Dos de ellos conforman la sociabilidad natural 
de los humanos cuando los dos otros fundamentan su insociabilidad. La conmiseración permite una identificación con el otro y una simpatía en el sentido etimológico de un sufrir-con, que, al generarnos tristeza, nos lleva a intentar suprimir las causas del sufrimiento del otro: "...la conmiseración; podemos definirla como una tristeza surgida del daño de otro." (Spinoza, 2014, III, 22, Escolio: 237). Además, la ambición de gloria nos permite también acercarnos al otro ya que, dado que el reconocimiento del cual esperamos la gloria solo puede provenir de los otros, para obtener gloria, debemos necesariamente someternos a los criterios comunes de valoración de las acciones. Tal como lo expresaba el Tratado de la reforma del entendimiento, quién busca la gloria y los honores está condenado a someterse a las reglas sociales, ya que son estas que determinan, in fine, lo que es digno de admiración o lo que merece honores: "Finalmente, el honor es un gran estorbo [para alcanzar el Bien supremo], ya que, para alcanzarlo, tenemos que orientar nuestra vida conforme al criterio de los hombres, evitando lo que suelen evitar y buscando lo que suelen buscar." (Spinoza, 1988, I, 5: 77) ${ }^{14}$. En ambos casos,

${ }^{14}$ En este tratado que se dirige a personas en búsqueda de sabiduría, el someterse a normas comunes está valorado de manera negativa, cuando, al contrario, en el campo de la política, está valorado de manera positiva. entonces, tanto la conmiseración como el deseo de gloria pueden explicar cierto acercamiento entre los hombres, lo cual podría dejarnos pensar en una sociabilidad natural de los seres humanos.

Sin embargo, los dos afectos restantes presentan la estructura exactamente contraria a la que acabamos de ver en la identificación positiva de la conmiseración y del deseo de gloria. El mismo mecanismo mimético que explica la conmiseración, permite entender cómo surge la envidia: "Si imaginamos que alguien goza de alguna cosa que solo uno puede poseer, nos esforzamos por conseguir que posea esa cosa." (Spinoza, 2014, III, 32: 248). La identificación con el otro que nos lleva a sufrir con él y por lo tanto a hacer todo lo posible para limitar este sufrimiento, es también lo que genera la envidia según la cual, en vista que es nuestro semejante, no hay ninguna razón por la cual él debería poder gozar de alguna cosa de la cual nosotros no podemos apoderarnos. De la misma manera, la ambición de dominación también proviene de este mismo mecanismo mimético, a raíz del cual cada uno de nosotros se esfuerza para que los otros vivan según nuestro criterio, porque la aceptación de estos por parte de otros nos confirma retroactivamente y miméticamente su validez: “... así vemos que cada cual por naturaleza, apetece que los demás vivan como él 
lo haría según su índole propia y como todos apetecen lo mismo, se estorban los unos a los otros y, queriendo todos ser amados o alabados por todos, resulta que se odian entre sí." (Spinoza, 2014, III, 31, Escolio: 248). Así, la ambición de dominación es el inverso de la ambición de gloria, tal como la envidia es el inverso de la conmiseración. Pero los cuatro afectos se basan en un solo mecanismo: el mimetismo.

Este único mecanismo mimético explica que exista un fenómeno fundamental de reciprocidad (Matheron, 1969) en las relaciones humanas, reciprocidad que comienza como una reciprocidad positiva y que termina en una reciprocidad negativa. En ciertas circunstancias los afectos pueden ser positivos y acercar los hombres los unos a los otros. Pero es justamente lo que los hace positivos que también genera la violencia interhumana, es decir la identificación con el otro y el mimetismo. Nadie mejor que René Girard explicó (Girard, 1983 \& Girard, 1985), en su teoría del deseo mimético, en qué medida y según qué lógica, el acercar a los hombres es también tomar el riesgo de alejarlos de manera violenta o más bien dicho cómo es el mismo mecanismo que une a los hombres que amenaza separarlos. Ya hemos analizado los vínculos entre Spinoza y Girard en otros trabajos (Vinolo, 2016), pero notemos aquí que ambos quieren restablecer la continuidad entre Estado político y Estado prepolítico y que ambos piensan esta continuidad gracias al juego del único mimetismo de los afectos. Efectivamente, bien entendemos que en primer término el acercar a los seres humanos pueda facilitar las relaciones sociales ya que, hasta cierto punto la semejanza es pacificadora. Si comparten valores, modos de vida, visiones del mundo y aspiraciones políticas, podemos pensar que les será mucho más fácil, a individuos, convivir en una sociedad pacificada. Tal como solemos pensarlo tradicionalmente, la violencia proviene de la diferencia o de la intolerancia a la diferencia. Sin embargo, tanto Spinoza como Girard nos permiten cuestionar este discurso infantil, dado que ambos muestran que la semejanza es aún más violenta que la diferencia. Efectivamente, ¿qué es la envidia sino el deseo de tener lo mismo que lo que el otro tiene y de preferir que nadie lo tenga a que lo tenga otra persona? De la misma manera, la ambición de dominación es un deseo que los otros vivan como nosotros y que piensen como nosotros. Una vez más, es la semejanza que genera violencia. Hay así una paradoja según la cual son los mismos afectos sociales que al acercar los seres humanos y al permitir una identificación de los unos con los otros, provoca el ciclo negativo de los afectos antisociales. 
Por esta razón, de estos dos ciclos de reciprocidad, siempre es el ciclo negativo el que es más fuerte. Por este motivo, Spinoza puede escribir que: "En la medida en que los hombres están sujetos a las pasiones, no puede decirse que concuerdan en naturaleza." (Spinoza, 2014, IV, 32: 344). Y bien entendemos por qué, ya que los afectos humanos nos transportan en un círculo vicioso del cual no podemos salir dado que se autoalimentan. Cuando imaginamos que algún ser semejante a nosotros sufre, sentimos conmiseración por él. Por este motivo, cuando compartimos el sufrimiento de alguien intentamos aliviarlo: "Nos esforzamos cuanto podemos por librar de su miseria a una cosa que nos mueve a conmiseración." (Spinoza, 2014, III, 27, Corolario 3: 242). Pero sí logramos atenuar su sufrimiento, él se alegra y por lo tanto nos alegramos de ser causa de esta felicidad. Estamos entonces en la gloria. Tercero, dado que el sentimiento de ser causa de la alegría de alguien es de por sí agradable queremos repetirlo y mantenerlo, lo que fundamenta el deseo de gloria. Sin embargo, aunque queramos la felicidad de los otros no podemos sacrificar nuestros intereses ni nuestros deseos; de allí que el deseo de gloria se transforme en ambición de dominación, ya que nos será más fácil satisfacer a los otros (y por lo tanto glorificarnos) si ya están de acuerdo con nuestros valores y nuestros modos de vida. Cuarto, si logramos convencer a los otros de que deben adoptar nuestros modos de vida y por lo tanto hacerles desear lo que nosotros mismos deseamos, intentarán apropiárselo y si lo logran, generará envidia de nuestra parte. En fin, si la lucha por la posesión de objetos que provoca la envidia nos es favorable, la desposesión del otro le generará tristeza y recaeremos en el primer momento del proceso en el cual alguien que sufre nos provoca conmiseración. Así, si nos limitamos a estos afectos, no hay salida posible del círculo de la violencia ya que la articulación: conmiseración, gloria, ambición de dominación, envidia es circular y por lo tanto infinita.

Sin embargo, en el Tratado Político Spinoza añade un último proceso afectivo que nos permite explicar el surgimiento inmanente del estado político: "Dado que los hombres se guían, como hemos dicho, más por la pasión que por la razón, la multitud tiende naturalmente a asociarse, no porque le guíe la razón, sino algún sentimiento común y quiere ser conducida como por una sola mente, es decir (como dijimos en el $\$ 9$ del capítulo III), por una esperanza o un miedo común o por anhelo de vengar un mismo daño." (Spinoza, 2013, VI, 1: 154). Lo que une a los hombres en última instancia es un afecto común: esperanza común, miedo común u odio común. Pero entre todos, el más importante es 
el miedo ya que en la lógica utilitarista de Spinoza, es el miedo el que puede impedir que los individuos se ataquen los unos a los otros y en ningún caso son valores. Tal como nos lo recuerda la Ética, los hombres renunciarán a hacerse daño los unos los otros bajo la única condición que perciban que les es beneficioso: "... cada cual se abstiene de inferir un daño a otro, por temor a un daño mayor." (Spinoza, 2014, IV, 37, Escolio II: 356). Sin embargo, el miedo común que une a los hombres en una misma sociedad tiene un límite cuantitativo, límite a partir del cual lo que une de repente desune y disuelve. Aparece otra vez la gran voluntad de continuidad de Spinoza en su filosofía. Cuando el miedo que pesa sobre los ciudadanos pasa cierto límite, se transforma en indignación y esta al contrario del miedo, lleva a la disolución del Estado:

Por consiguiente, para que la sociedad sea autónoma, tiene que mantener los motivos del miedo y del respeto; de lo contrario, deja de existir la sociedad. Pues, para aquellos o aquel que detenta el poder del Estado, es tan imposible correr borracho o desnudo con prostitutas por las plazas, hacer el payaso, violar o despreciar abiertamente las leyes por él dictadas y, al mismo tiempo, mantener la majestad estatal, como lo es ser $y$, a la vez, no ser. Asesinar a los súbditos, espoliarlos, raptar a las vírgenes y cosas análogas transforman el miedo en indignación y, por tanto, el estado político en estado de hostilidad. (Spinoza, 2013, IV, 4: 144-145).

Tenemos así, en el fundamento afectivo del estado político un juego cuantitativo entre miedo común e indignación, que nos permite pensar a la vez la construcción y la disolución del estado político. Este punto nos ayuda a pensar el surgimiento del Estado porque, en Spinoza, es el mismo mecanismo que permite explicar a ambos fenómenos de disolución y de construcción, porque nunca se puede disolver completamente la sociedad, sino únicamente transformar ${ }^{15}$. Por lo cual la indignación que pone en peligro al Estado, en realidad crea otro. En el texto que mencionamos previamente (Spinoza, 2013, VI, 1: 154), Spinoza hacía referencia a otro texto en el cual aparece la verdadera clave del

${ }^{15}$ Esta idea según la cual la disolución de un Estado es la creación de otro, por lo que nunca es una destrucción simple y radical, está claramente expresada por Spinoza: "Nunca sucede, pues, que, a consecuencia de las discordias y sediciones que surgen a menudo en la sociedad, los ciudadanos la disuelvan (como acontece con frecuencia en otras asociaciones). Simplemente, cambiarán su forma por otra, si es que las desavenencias no se pueden superar manteniendo la misma estructura de la sociedad." (Spinoza, 2013, VI, 2: 155) 
surgimiento del estado político, el afecto que permite entender todo el proceso, la indignación: "Hay que considerar en tercer y último lugar, que cuanto provoca la indignación en la mayoría de los ciudadanos es menos propio del derecho de la sociedad." (Spinoza, 2013, III, 9: 134). Y en un segundo texto aún más preciso, es otra vez la indignación que explica la disolución del Estado y sobre todo el momento en el cual el miedo común que inspira el Estado, se transforma en indignación:"Pero, si esas leyes son de tal índole que no puedan ser infringidas, sin que con ello se debilite la fortaleza de la sociedad, es decir, sin que el miedo de la mayor parte de los ciudadanos se transforme en indignación, la sociedad se disuelve automáticamente..." (Spinoza, 2013, IV, 6: 146-147). Debemos entonces entender el funcionamiento de la indignación ya que es este afecto el que al final permite entender el surgimiento del estado político.

Notemos primero, para no introducir rupturas cualitativas, que la indignación es también un afecto mimético: "Llamaremos [...] indignación, al odio hacia aquel que ha hecho mal a otro." (Spinoza, 2014, III, 22, Escolio: 237). Pero, sobre todo, lo que explica la diferencia entre el miedo común que une a los seres humanos bajo la autoridad de un Estado y la indignación que es la fuente de su disolución, es el carácter público de esta. Si el miedo común une y la indignación disuelve, es porque en el caso del miedo, cada uno de los ciudadanos se encuentra solo y por lo tanto impotente frente al Estado, lo que limita su capacidad a rebelarse. Se debe distinguir entre lo común y lo público en este sentido que, en el caso de lo público, además de compartir un afecto, sabemos que los otros lo sienten también cuando el miedo común, si bien es compartido, se experimenta en la soledad del individuo. A la diferencia del miedo común, el hecho que la indignación sea pública informa a todos los ciudadanos que sienten un afecto común y que por lo tanto pueden unirse para luchar en contra del poder que es claramente identificado y mostrado como un enemigo común. Además, este afecto público, al mostrarse, se autoalimenta, se multiplica y se disemina por la ley fundamental del mimetismo.

Es con este mecanismo de la indignación que podemos explicar cómo surgen los Estados tal como lo indica Matheron (2011: 212-214), sustituyendo la soledad de los individuos frente al tirano, a la soledad de los individuos en el estado de naturaleza. Para esto, hay que añadir un último punto acerca de la indignación. El mimetismo de los afectos se aplica de manera prioritaria a los individuos que percibimos como semejantes a nosotros. Si dos individuos experimentan dos afectos diferentes 
frente a nosotros, el afecto que nosotros sentiremos es el del individuo que percibimos como más semejante a nosotros: "... sentimos conmiseración no solo hacia la cosa que amamos, sino también hacia aquella sobre la que no proyectamos con anterioridad afecto alguno, con tal que la juzguemos semejante a nosotros..." (Spinoza, 2014, III, 22, Escolio: $237)^{16}$. Esto no se aplica únicamente a la conmiseración, ya que lo encontramos en la ley fundamental de toda la afectividad humana:"... si imaginamos a alguien semejante a nosotros experimentando algún afecto, esa imaginación expresará una afección de nuestro cuerpo semejante a ese afecto $y$, de esta suerte, en virtud del hecho de imaginar una cosa semejante a nosotros experimentando algún afecto, somos afectados por un afecto semejante al suyo." (Spinoza, 2014, III, 27, demo: 241-242) ${ }^{17}$. Así, si bien la ley de la afectividad interhumana es el mimetismo, la semejanza es el verdadero catalizador de esta ley universal.

\section{III - DURACIÓN Y MORALIDAD DEL ESTADO - LA INDIGNACIÓN SOCIALIZANTE}

Disponemos ahora de todos los elementos para pensar el estado político en Spinoza: la necesidad de un surgimiento afectivo, la ley fundamental de la

${ }^{16}$ [Subrayamos]

17 [Subrayamos] afectividad humana, cuatro afectos que se reparten entre reciprocidad positiva y reciprocidad negativa, la indignación y la semejanza como catalizador del mimetismo. Gracias a estos puntos podemos entonces pensar el estado de naturaleza y su necesario devenir político. Imaginemos seres humanos en el estado de naturaleza. Si alguno de ellos sufre, sea por enfermedad, falta de comida o simplemente por la dureza de vivir en este estado, ya sea por conmiseración o por gloria, los otros le ayudarán y lucharán para aliviar sus sufrimientos. Sin embargo, una vez este sufrimiento es eliminado, estos afectos se cambiarán en deseo de gloria, ambición de dominación y en envidia. Lo que era un acercamiento entre los individuos se transforma en violencia y la persona que fue ayudada en un primer momento será ahora atacada y violentada. Sin embargo, al ver esos ataques otros individuos se sentirán indignados y este sentimiento se diseminará entre todos los individuos. En cada agresión, entonces, todos los individuos estarán involucrados miméticamente en la lucha. Pero, tal como lo hemos visto, cada uno siente prioritariamente el afecto de quién le es más semejante y por lo tanto lucha a su lado. Por lo que cada individuo lucha en contra de quién le es menos idéntico. Podemos así entender cómo surgen normas comunes sin que haya ningún acuerdo contractual, por 
simple mimetismo. Dado que cada uno lucha al lado de quién le es más semejante, se crean mayorías que se indignan cada vez más en contra de las personas que les son menos semejantes. Y poco a poco por repetición de este mecanismo, la lucha de todos contra todos se transforma en una lucha de todos contra pocos y de todos contra uno, que es la forma más primitiva de la creación y de unión de los colectivos humanos ${ }^{18}$.

A medida que las personas que se alejen de las normas comunes sean eliminadas, se crea el poder de la multitud (y del Estado ${ }^{19}$ ) que se asimila a su potencia y por lo tanto a su derecho. Con la creación de la potencia de esta multitud, es decir de un grupo mayoritario que, por mimetismo de los afectos, tiende a luchar siempre del lado de las personas que les son más semejantes, todo el mundo temerá que esta potencia pueda llegar a violentarle si se alejan de las normas comunes. Así, la creación mimética de este poder de la multitud permite entender que los individuos ya no se teman los unos a los otros, sino que todos teman a lo mismo, precisamente a

\footnotetext{
${ }^{18}$ Una vez más sobre este punto podemos notar la proximidad de Spinoza y de Girard, asumiendo que el surgimiento del orden colectivo está basado últimamente en una violencia colectiva, es decir en un linchamiento.

19 "Este derecho que se define por el poder de la multitud suele denominarse Estado." (Spinoza, 2013, II, 17: 118)
}

la potencia de la multitud. Lo que era un miedo individual de todos en el estado de naturaleza se transformó en un miedo común de una misma entidad, lo que es la marca del estado político: ". ..en el estado político todos temen las mismas cosas y todos cuentan con una y la misma garantía de seguridad y una misma razón de vivir." (Spinoza, 2013, III, 3: 128). La indignación mimética permite entender cómo surge de manera inmanente el estado político, sin hacer referencia a un contrato, a valores trascendentes ni a una esencia humana. El surgimiento del Estado proviene entonces de un movimiento conservador ${ }^{20}$ según el cual los afectos circulan más fácilmente entre personas que para bien o para mal, se identifican más o menos los unos con los otros.

Pero para entender este fenómeno, se debe precisar que la indignación en Spinoza, no está basada en valores cualitativos ni en un deber de desobediencia, que se podría justificar por la existencia de alguna legitimidad por encima de la legalidad. El proceso creador y regulador del Estado, por provenir de la indignación es exclusivamente cuantitativo y por lo tanto moralmente neutro. Tal como lo mostró Charles Ramond (2016), las normas comunes emergentes se basan en la

${ }^{20}$ Algunos autores investigaron la relación de Spinoza con cierto conservatismo. Cf. (Zourabichvili, 2002) 
única cantidad de sus promotores. De allí la insistencia cuantitativa de Spinoza a la hora de determinar a partir de cuándo la indignación amenaza al Estado o a partir de cuándo el miedo común se transforma en indignación pública, lo que genera a la vez la destrucción de la forma existente del orden político y la creación de uno nuevo: "Hay que considerar en tercer y último lugar, que cuanto provoca la indignación en la mayoría de los ciudadanos es menos propio del derecho de la sociedad. [...]Y Y como el derecho de la sociedad se define por la potencia conjunta de la multitud, está claro que el derecho y la potencia de la sociedad disminuye en cuanto ella misma da motivos para que muchos conspiren lo mismo." (Spinoza, 2013, III, 9: 134)21. Sin embargo, ya que la indignación amenaza siempre a toda forma política, ¿cómo explicar la perseverancia en su ser del orden político?

Efectivamente, si es el único miedo común que garantiza la unión de los ciudadanos, dado que la frontera entre miedo común e indignación es muy inestable (por ser cuantitativa), la sociedad está siempre bajo la amenaza de ser destruida por la indignación de un grupo cada vez más grande de ciudadanos. Por esta razón, la mejor de las sociedades cual sea su forma política (aristo-

${ }^{21}$ [Subrayamos y corregimos la traducción de Domínguez para restablecer la "potencia" en vez del "poder"] cracia, monarquía o democracia), tiene que producir efectos que nos permitan entender en qué medida los ciudadanos deseen positivamente formar parte de esta. Tal como lo vemos en el párrafo 19 del capítulo II del Tratado Político, para que una sociedad dure no basta que los ciudadanos respeten las leyes por miedo, sino que haya cierto obsequium que guía a los ciudadanos. Esta palabra obsequium está traducida por Domínguez con el concepto de "obediencia": "...obediencia es la voluntad constante de ejecutar lo que es bueno según derecho y que, por unánime decisión, debe ser puesto en práctica." (Spinoza, 2013, II, 19: 121). Sin embargo, dado que esta obediencia es el fruto de una "voluntad constante", podríamos en realidad traducirlo por "consentimiento" más que "obediencia", si aceptamos oponer la obediencia como sumisión a una ley externa y el consentimiento como la sumisión a una ley interna o internalizada. Más aún, según un punto de vista derridiano, se podría cuestionar esta oposición entre obediencia externa y consentimiento, porque toda obediencia, incluso la que podría parecer más externa, es en realidad una forma de consentimiento ya que obedecer siempre es consentir a obedecer. Tal como lo vemos en los ejemplos de resistencias, uno siempre puede negarse a obedecer hasta la muerte, por lo que obedecer siempre es 
consentir a obedecer. Por lo tanto, el mejor de los Estados es el que más fácilmente produce obediencia positiva, es decir consentimiento. De la misma manera, en el párrafo 23 del capítulo II del mismo libro, Spinoza afirma que la justicia que produce el Estado no puede limitarse a una justicia externa, a un respeto forzado de las leyes positivas del Estado. La justicia es también una aceptación interna, en este sentido que: "... se dice justo aquel que tiene una voluntad constante de dar a cada uno lo suyo, e injusto, por el contrario, aquel que se esfuerza en hacer suyo lo que es de otro." (Spinoza, 2013, II, 23: 124). Tal como para la obediencia, la verdadera justicia implica una interiorización de las reglas positivas del Estado y la voluntad constante de cumplirlas. Por lo cual podemos concluir que, si bien el Estado no surge de un proceso moral, el que más puede perseverar en su ser es aquel que produce efectos que tienen la forma de una conciencia moral (Matheron, 2011(3): 191), en este sentido que, en un Estado correctamente formado, cada ciudadano sentirá el deseo positivo de obedecer a sus leyes: "...el Estado que pone su máximo empeño en que los hombres sean conducidos por el miedo carecerá más bien de vicio que poseerá virtud." (Spinoza, 2013, X, 8: 276).

Sin embargo, este proceso supone que el soberano no emita cualquier ley o decreto, ni se deje llevar por sus únicos deseos. Hay cosas que no puede exigir de los ciudadanos un soberano sin ponerse en peligro: "En vano mandaría a un súbdito que odiara a quien le hizo un favor y amara a quien le hizo daño, que no se ofendiera con las injurias, que no deseara librarse del miedo y muchísimas otras cosas similares que se derivan necesariamente de las leyes de la naturaleza humana." (Spinoza, 2012, XVII, 1: 353). Hay así un límite a la potencia soberana, pero este límite es plenamente inmanente y a posteriori. Si un soberano emite leyes absurdas, maximiza sus posibilidades chances de generar indignación y por lo tanto minimiza sus chances de durar. Entendemos entonces en qué medida la política plenamente basada en un surgimiento afectivo, es el mecanismo que simula la racionalidad. Los gobernantes para poder durar, están obligados a no alejarse demasiado de la racionalidad, dado que es la racionalidad (o lo que más se acerca de ésta) que puede sobrepasar la singularidad de los afectos que siente cada ser humano. Lo más probable para garantizar que se indigne el mínimo posible de ciudadanos, es de emitir leyes que no se alejen mucho de lo racional, ya que es probable (aunque no esté seguro) que la mayoría está cerca de una adecuación con estas posiciones. Por eso, dado que compartimos la racionalidad cuando los afectos son singulares, el hecho de tomar las decisiones en gran número 
maximiza las chances de acercarnos a la razón: "Pues, como los malos sentimientos arrastran a los hombres en distintas direcciones, solo cuando estos desean lo honesto o lo que, al menos, lo parece, pueden ser guiados como por una sola mente." (Spinoza, 2013, VIII, 6: 215).

Pero este proceso de racionalización no debe pensarse desde un punto de vista finalista según el cual la función del Estado es de racionalizar a los ciudadanos. Sería reintroducir finalidad en la filosofía, lo que Spinoza no puede aceptar. Al contrario, el proceso de racionalización se da de hecho, por el simple hecho que solo pueden durar los regímenes que manifiestan un mínimo de racionalidad. Todos los otros serán destruidos por la indignación de la mayoría de los ciudadanos. De allí la valoración sumamente positiva de la democracia en Spinoza, no por razones axiológicas, no en nombre de los valores de la democracia, sino por su capacidad de durar, donde se multiplican los electores y por lo tanto se maximizan sus chances de no alejarse demasiado de la racionalidad: "Añádase a lo anterior que tales absurdos son menos de temer en un Estado democrático; es casi imposible, en efecto, que la mayor parte de una asamblea, si esta es numerosa, se ponga de acuerdo en un absurdo." (Spinoza, 2012, XVI, 3: 342). Dado que la indignación es el autoregulador inmanente de las sociedades políticas, se ga- rantiza de hecho que las sociedades que perseveran en su ser están guiadas por un mínimo de racionalidad, por lo que la política obliga a los gobernantes a actuar como si fuesen racionales.

\section{CONCLUSIÓN}

Entendemos ahora en qué medida la indignación es el núcleo de la política en Spinoza, y cómo se debe repensar este concepto para que pueda desplegar toda su eficiencia dentro del campo de los afectos y de lo político. Si la ley fundamental de los afectos es el mimetismo, podemos ver tanto con Spinoza como con Girard que el primer momento de reciprocidad positiva siempre está sobrepasado por un proceso de reciprocidad negativa. Por lo que es un solo y único mecanismo que explica el surgimiento del Estado, sus transformaciones, así como su perseverancia en el ser. Primero, la indignación permite el surgimiento inmanente del estado político por el simple contagio mimético. Dado que el mimetismo se basa en la identificación con el otro y que uno se identifica más fácilmente, en Spinoza, con quién le es más semejante, normas comunes emergen por exclusión de los que están más alejados de las ideas mayoritarias.

Por repetición mimética, la guerra de todos contra todos se transforma de manera evolutiva en una lucha de todos contra algunos y finalmente de 
todos contra uno. El primer momento de la política tiene entonces la forma de un linchamiento ${ }^{22}$ conservador, que nos permite pensar que no hay ruptura cualitativa entre el estado de naturaleza y el estado político, sino que todo sucede según la continuidad del mimetismo de los afectos que nos hacen pasar de la reciprocidad positiva de la identificación, a la reciprocidad negativa y violenta de la envidia y de la ambición de dominación. Segundo, es a raíz de este primer momento que puede surgir el miedo común a la potencia de la multitud, miedo que garantiza la unidad de la sociedad y consecuentemente su orden. Al miedo de todos frente a todos que amenaza el estado de naturaleza, sucede el miedo de todos por la misma potencia de la multitud que han visto actuar en el linchamiento originario. La primera unificación de la sociedad es entonces negativa, en el doble sentido que surge de un afecto negativo -la indignación- y además se mantiene por otro afecto negativo -el miedo común. Sin embargo, este mecanismo de la indignación no desaparece con la formación del estado político; al contrario, permanece en todas las sociedades como autoregulador social.

Efectivamente, las sociedades solo pueden mantenerse con la condi-

22 « [...] : la forme élémentaire de la démocratie, selon Spinoza, c'est le lynchage. » (Matheron, 2011(1): 228) ción de no generar demasiada indignación entre los ciudadanos. Caso contrario, la indignación será tan fuerte que ningún gobernante podrá perseverar en su ser a largo plazo. Pero esta indignación como regulador social no se basa en valores que podríamos oponer a los de las leyes positivas. En el híper-racionalismo de Spinoza, todo es una cuestión de cantidad. Una misma actitud del gobernante será aceptada hasta cierto punto, pero pasado este punto generará la indignación que marcará el fin de su reino ${ }^{23}$. Así, la indignación explica el surgimiento del Estado y también su perseverancia en su ser de manera evolucionista, en este sentido que su simple perseverar en el ser evidencia cierta racionalidad de este. Por esta razón, el carácter necesario de la política en Spinoza no se debe entender como una necesidad moral del deber-ser, ni de una finalidad antropológica, menos aún de una necesidad que surge de una negociación contractual. Tal como la necesidad que yace en la Ética es una necesidad fría, neutra y por lo tanto violenta.

${ }^{23}$ En este sentido, Charles Ramond nota que, si bien puede parecer indignante a muchos pueblos que, tal como lo dice Spinoza, los Príncipes rapten a las vírgenes (Spinoza, 2013, IV, 4: 115), fue durante muchos siglos un modelo valorizado positivamente en el imaginario popular, con el paradigma del Príncipe azul (Ramond, 2016: 420). Así el rapto de vírgenes es de vez en cuando indignante, de vez en cuando deseado, es decir aceptable hasta cierto punto que marca el momento de la indignación. 
Es la necesidad de una pura descripción del ser (en oposición a la imposición de un deber-ser): dado lo que son los afectos humanos, no puede no surgir un Estado.

Entendemos entonces que la política inmanente de Spinoza sea decepcionante para quienes esperan de esta que les diga cómo se debe vivir, según qué valores y con qué objetivos, ya que la indignación exclusivamente cuantitativa se da como axiológicamente neutra. La política de Spinoza, al describirnos el surgimiento del Estado de manera mimética, violenta e inmanente no se da como objetivo el describir el deber-ser de los colectivos humanos, sino únicamente el explicar cómo se crean y perseveran en su ser. Sin embargo, esta decepción se puede atenuar para quién acepta que, tal como lo nota Vidal Peña en la introducción de su traducción de la Ética, no hay ninguna razón por la cual debamos esperar de un filósofo que sea simpático ${ }^{24}$, no es su papel, ni debería ser su aspiración.

24 "Podrá ser muy explicable que, en nuestro tiempo, el pensamiento de Espinoza resulte antipático (e incluso podrían buscarse explicaciones, más o menos psicoanalíticas, para esa complacencia en la antipática dureza), pero, con independencia de que pueda haber aún gentes que aprecien esa lucidez irreductible al halago, no parece correcto tergiversar ese pensamiento para volverlo simpático.", Vidal Peña, introducción a la Ética de Spinoza, in Spinoza, Ética, Alianza Editorial, Madrid, 1987, 2014, p. 47

\section{BIBLIOGRAFÍA}

Aristóteles. (1988), Política. Madrid: Gredos.

Aristóteles. 1985, (1988(1)), Ética a Nicomáquea. Madrid: Gredos.

Dupuy, J-P. (2002), Avions-nous oublié le mal? Penser la politique après le 11 septembre. Paris: Bayard.

Girard, R. (1983), La violencia y lo sagrado. Barcelona: Anagrama.

Girard, R. (1985), Mentira romántica y verdad novelesca. Barcelona: Anagrama.

Girard, R. 1999, (2001), Je vois Satan tomber comme l'éclair. Paris : Grasset.

Hobbes, T. (1982), Leviatán, Bogotá: Editorial Skla.

Matheron, A. (1969), Individu et communautéchez Spinoza. Paris : Éditions de minuit.

Matheron, A. (2011), Le problème de l'évolution de Spinoza, in Études sur Spinoza et les philosophes de l'âge classique. Lyon : ENS Éditions, pp. 205-218.

Matheron, A. (2011(1)), L'indignation et le conatus de l'État spinoziste, in Études sur Spinoza et les philosophes de l'âge classique. Lyon : ENS Éditions, pp. 219-229.

Matheron, A. (2011(2)), Éthique et politique chez Spinoza, in Études sur Spinoza et les philosophes de l'âge classique. Lyon : ENS Éditions, pp. 195-203. 
Matheron, A. (2011(3)), État et moralité chaez Spinoza, in Études sur Spinoza et les philosophes de l'âge classique. Lyon: ENS Éditions, pp. 185-193.

Peña, V. (2011), Introducción a la Ética de Spinoza, in Spinoza, Ética, Madrid: Alianza Editorial, pp. 9-47.

Ramond, C. (1995), Qualité et quantité dans la philosophie de Spinoza. Paris : PUF.

Ramond, C. (2016), Vers une démocratie sans valeurs?, in Charles Ramond, Spinoza contemporain, Philosophie, Éthique, Politique. Paris: L'Harmattan, pp. 405-421.

Spinoza, 1986, (2013), Tratado Político. Madrid: Alianza Editorial.

Spinoza, 1986, (2012), Tratado Teológico-Político. Madrid: Alianza Editorial.

Spinoza, 1987, (2014), Ética. Madrid, Alianza Editorial.

Spinoza, (1988), Tratado de la reforma del entendimiento. Madrid: Alianza editorial.

Spinoza, (2007), Epistolario. Buenos Aires: Colihue Clásica.

Terrel, J. (2001), Les théories du pacte social, Droit naturel, souveraineté et contrat de Bodin à Rousseau. Paris : Seuil.

Vinolo, S. (2005), René Girard, Du mimétisme à I'hominisation, "La vio- lence différante ». Paris : L’Harmattan.

Vinolo, S. (2007), René Girard, Épistémologie du sacré, "En Vérité je vous le dis ». Paris: L'Harmattan. 
